\title{
3D numerical transport study of the edge ergodized plasma in TEXTOR-DED
}

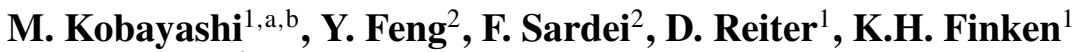 \\ and D. Reiser ${ }^{1}$ \\ ${ }^{1}$ Institut für Plasmaphysik, Forschungszentrum Jülich GmbH, EURATOM Association, \\ Trilateral Euregio Cluster, D-52425 Jülich, Germany \\ ${ }^{2}$ Max-Planck-Institut für Plasmaphysik, EURATOM Association, D-17491 Greifswald, \\ Germany \\ E-mail: masahiro@nifs.ac.jp
}

Received 11 November 2003, accepted for publication 25 February 2004

Published 28 May 2004

Online at stacks.iop.org/NF/44/S64

doi:10.1088/0029-5515/44/6/S07

\begin{abstract}
Transport properties of the edge ergodized plasma in TEXTOR-DED (Finken K H and Wolf G H 1997 Fusion Eng. Des. 37 337) are studied using the 3D plasma edge transport code package, EMC3-EIRENE, prior to the forthcoming experimental campaign. EMC3 solves the steady-state plasma fluid equations of mass, momentum and energy, in almost any arbitrary 3D magnetic and bounding surface configurations. It is iteratively coupled with EIRENE, a 3D kinetic neutral particle transport code. Two different levels of ergodization have been selected in order to assess the additional effects of ergodicity on edge plasma transport. The code predicts a significant change in the radial profile of electron temperature for the higher ergodization and a pronounced correlation between the plasma flow fields and the magnetic field structure. The role of the (outermost) 'laminar zone' on the energy deposition pattern onto the divertor is analysed. It is found that its influence depends sensitively on the degree of ergodization achieved.
\end{abstract}

PACS numbers: 52.55.Fa, 52.65.Pp

\section{Introduction}

It has been clearly demonstrated, e.g. in Tore Supra (ED) [1], that externally applied perturbations of the confining magnetic field, which are localized in the tokamak edge region, can actively influence particle and heat exhaust. Along these lines the new TEXTOR dynamic ergodic divertor (DED) [2] has also been proposed as a potential method of controlling the edge plasma transport with external resonant magnetic perturbations. Therefore, transport properties in the edge ergodized plasma in the DED configuration are analysed by a $3 \mathrm{D}$ edge fluid code package, prior to the forthcoming experimental campaign, in order to assess the predictive capability of these edge models in such geometries.

The coils for the DED perturbation field are installed at the high field side of the machine with the mode $(m, n)=(12,4)$, designed to be resonant with the $q=3$ magnetic flux surface. The side bands of the main mode create subsequent island

\footnotetext{
a Author to whom any correspondence should be addressed.

b Current address: Theory and data analysis division, National Institute for Fusion Science 322-6 Oroshi, Toki 509-5292, Japan.
}

chains. Thus, an ergodic region at the edge of the plasma is created by the overlap of the island chains of different modes. The coils are energized with independent phases relative to each other, so that a rotation of the perturbation field can be excited by selecting a proper phase. This is a unique feature of the DED, which is not present in conventional ergodic divertors. The aim of the rotation is to smear out the localized heat load pattern on the divertor and to induce a plasma rotation, which is expected to lead to confinement improvement. In this paper, however, the object of the study is a static, partially ergodic field, where many physical aspects still remain unknown.

The ergodization of the magnetic field will change the transport characteristics due to magnetic flutter. This has been one of the major topics in plasma transport theory in the last few decades. Probably, the best known result is the paper by Rechester and Rosenbluth in the 1970's [3]. They proposed an electron heat transport model in a fully ergodic magnetic field region by taking into account a 'braiding' effect of the field lines, which gives a significantly enlarged effective cross-field transport for electron energy. In ergodic divertors this may result in a flattening of the electron edge 
temperature profiles because of the very large intrinsic parallel heat conductivity when the degree of ergodization is high enough. Experimental examples of such flattening are found in Tore Supra, Text and JFT-2M [4]. The 3D transport modelling of the heat conduction equation in the ergodic region of Tore Supra with the ERGOT1,2 codes reproduced such flattening and showed a good agreement of the obtained effective crossfield heat conductivity, $\chi_{\mathrm{erg}}$, with the expected values from the Rechester-Rosenbluth model [5]. Based on a statistical treatment of the field lines, i.e. employing the concept of field line diffusion coefficients, the nature of the transport in the ergodic region is also considered to be diffusive. On the other hand, in the region very close to the material wall surfaces, the magnetic field behaviour can certainly not be described as diffusive due to the smallness of the field line connection length, caused by the intersection of field lines with the wall. Such regions are referred to as 'laminar regions' in this paper (note: other definitions of the term 'laminar region' are also in use: see below and [4]). Here, the transport is expected to be dominated by parallel convection rather than radial diffusion. For example, in the DED the connection length of the field lines in the laminar region is of the order of several tens of metres, which is comparable with the scrape off layer (SOL) length of the axi-symmetric divertor tokamak. As the laminar region dominates, from a purely geometric point of view, in the outer edge region, one might expect that it will play an important role in the energy and particle deposition onto the wall. An isolated modelling of the laminar region was carried out using a simplified 2D transport code (finite element method) [6]. The model showed a certain correlation between the connection length and plasma parameter profiles, and the energy deposition patterns on the wall surfaces were also obtained.

However, these two topologically distinct edge regions, in realistic magnetic configurations, have no clear boundary on which to prescribe boundary conditions for such an isolated model. This is because of the definition of the laminar region, which, furthermore, is somewhat ambiguous. One might consider, at least, two definitions for this domain: One is the region where the connection length, $L_{c}$, is much smaller than the Kolmogorov length, $L_{K}$, i.e. $L_{c} \ll L_{K}$, in terms of the flux tube topology. Another possibility is to define it as that region in which the collisional mean free path, $\lambda$, is of the order of $L_{c}, L_{c} \sim \lambda$, or even longer in terms of transport. Although $L_{c}$ and $L_{K}$ are well defined for any given magnetic configuration, as, e.g. that studied in $[6,7]$ for TEXTOR$\mathrm{DED}$, the field line changes its connection length in a very complicated manner towards the edge. On the other hand, $\lambda$ is a function of the plasma parameters, and, thus, it may change significantly depending on the operational regime and the particular location of interest. In this sense, the transition between the two regions is difficult to specify clearly, and hence the modelling with a clear separation of the two regions always introduces uncertainty, especially in a proper boundary condition to connect these regions. Additionally, as seen later, magnetic islands appear at the inner radius or even at the very edge of the computational domain (the edge plasma region) if the ergodization is not complete. Because of these the transport process may further be modified around the islands.

Since the magnetic field structure in the ergodic divertor SOL is, in most cases, a mixture of these three different topologies, one inevitably needs 3D plasma fluid modelling tools, properly taking into account all these kinds of field line structures: closed flux surfaces, magnetic islands, stochastic sub-domains and the laminar region. The first such 3D fluid approach for TEXTOR was carried out by a Monte Carlo fluid simulation procedure for the electron heat conduction equation [8]. The E3D code used there demonstrated the feasibility of the 3D computation of the plasma fluid equations in partially ergodic domains. Extending this work, and for further quantification of some relevant edge physics terms one also needs to include convection terms as well as neutral transport in a self-consistent manner, because these can be expected to play an important role, particularly in the laminar region.

In this paper, the 3D fluid code, EMC3 [9], based on a similar Monte Carlo solution procedure, is applied to the DED plasma edge configuration, again for a static operational scenario. EMC3 has initially been developed as a fully 3D edge transport code for the stellarators W7-AS and W7-X [10,11]. The code solves the plasma fluid equations of mass, momentum and electron and ion energy, coupled self-consistently, and in almost any arbitrary magnetic and wall surface geometry. The recycling of neutrals from the walls is taken into account by coupling it with the 3D EIRENE code [12], again a Monte Carlo procedure, but, distinct from E3D and EMC3, a kinetic rather than a fluid transport code. The partially or even fully ergodic magnetic field topology is recovered in EMC3 using an accurate and fast mapping technique (reversible field line mapping, RFLM [9]). Supplemented with proper boundary conditions the code package provides a steady-state 3D plasma state: electron and ion temperature, particle density, flow field, atom and molecule densities, re-ionization source distributions, etc. In section 2, the algorithm of EMC3 is described. In section 3 , the degree of ergodization of the DED and its effects on the radial transport are discussed. In section 4 , a correlation between the resulting plasma parameter profiles and the magnetic field topology is discussed. In section 5, we investigate the energy and particle deposition process onto the divertor plate taking into account the full flux tube topology in the TEXTOR-DED SOL. Finally, in section 6, these issues are summarized.

\section{Modelling of the edge transport}

\subsection{Model equations}

EMC3 solves the steady-state plasma fluid equations in a 3D space. One can write the fluid equations in steady state as follows [13]:

$$
\nabla_{\|} \cdot\left(n \boldsymbol{V}_{\|}\right)+\nabla_{\perp} \cdot\left(-D \nabla_{\perp} n\right)=S_{p}
$$

$$
\begin{aligned}
& \nabla_{\|} \cdot\left(m_{i} n \boldsymbol{V}_{\|} \boldsymbol{V}_{\|}-\eta_{\|} \boldsymbol{\nabla}_{\|} \boldsymbol{V}_{\|}\right) \\
& \quad+\nabla_{\perp} \cdot\left(-m_{i} \boldsymbol{V}_{\|} D \nabla_{\perp} n-\eta_{\perp} \boldsymbol{\nabla}_{\perp} \boldsymbol{V}_{\|}\right)=-\nabla_{\|} p+S_{m} \\
& \begin{aligned}
\nabla_{\|} & \cdot\left(-\kappa_{i} \boldsymbol{\nabla}_{\|} T_{i}+\frac{5}{2} n T_{i} \boldsymbol{V}_{\|}\right)+\nabla_{\perp} \cdot\left(-\chi_{i} n \nabla_{\perp} T_{i}-\frac{5}{2} T_{i} D \nabla_{\perp} n\right) \\
\quad & =k\left(T_{e}-T_{i}\right)+S_{\mathrm{ei}}, \\
\nabla_{\|} \cdot & \left(-\kappa_{e} \nabla_{\|} T_{e}+\frac{5}{2} n T_{e} \boldsymbol{V}_{\|}\right)+\nabla_{\perp} \cdot\left(-\chi_{e} n \nabla_{\perp} T_{e}-\frac{5}{2} T_{e} D \nabla_{\perp} n\right) \\
\quad & =-k\left(T_{e}-T_{i}\right)+S_{\mathrm{ee}},
\end{aligned}
\end{aligned}
$$


where ' $\|$ ' and ' $\perp$ ' denote a direction parallel and perpendicular, respectively, to the magnetic field, $p=n\left(T_{i}+T_{e}\right)$ and $\eta_{\perp}=m_{i} n D$. The parallel transport coefficients, $\eta_{\|}, \kappa_{i}, \kappa_{e}$, are assumed to have their classical value and their non-linear dependence on the plasma parameters is dealt with by an iteration procedure. Cross-field transport coefficients, $D$, $\chi_{i}$ and $\chi_{e}$, which are the same for both directions normal to the field lines, have been set to constant values over the whole domain in our simulations described here. Through the iterative coupling with EIRENE, the interaction with the neutrals is taken into account via the terms $S_{p}, S_{m}, S_{\mathrm{ei}}$ and $S_{\text {ee }}$, which are sources/sinks of particles due to ionization, momentum due to charge exchange, and ion and electron energy due to ionization and charge exchange, respectively. Further details of the algorithm and the benchmarking of the code using both simple models and conventional 2D edge codes are described in [14-16].

\subsection{Plasma configuration and grid}

Figure 1 shows the configuration of the plasma and the computational domain for the present calculations in a poloidal cut at $\phi$ (toroidal angle) $=0^{\circ}$. In order to enhance the effect of the DED, the plasma was shifted inward (towards the HFS) with a vertical field of $200 \mathrm{G}$, and the ALT limiter was retracted, so that the plasma touches only the divertor at the HFS. This inward shifted configuration is also planned in the experiments. The toroidal extent of the domain is onefourth of the full torus, i.e. from $\phi=-22.5^{\circ}$ to $67.5^{\circ}$, because of the fourfold toroidal symmetry of the DED perturbation field. The radial extent, indicated by 'plasma' in the figure, represents the computational domain for the plasma, where the inner boundary was chosen from the closed magnetic flux surface, and the outer one from a flux surface of a nonperturbative case a few centimetres away from the divertor edge. Certain total particle and energy fluxes are specified

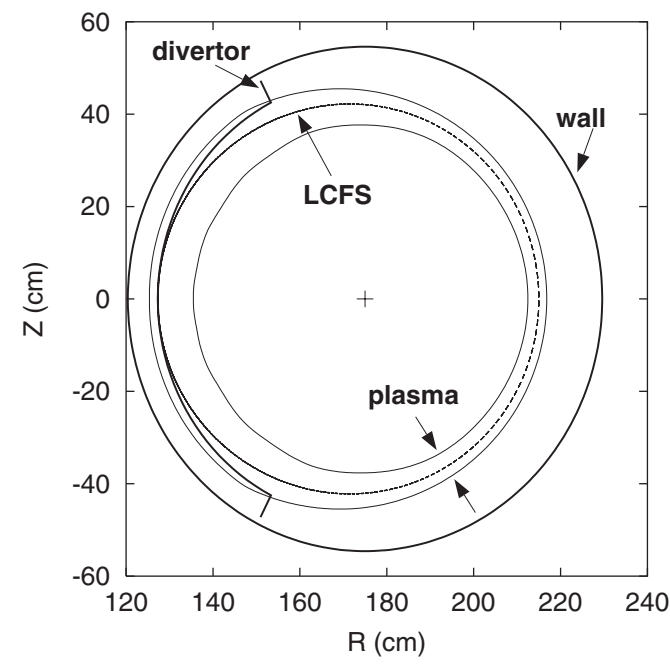

Figure 1. The configuration of the plasma and the computational domain in the poloidal cross section. The 'plasma' indicates the computational domain for the plasma while the domain for EIRENE is extended up to the 'wall'. The inner boundary is a magnetic flux surface. The LCFS of the non-perturbative case is also indicated in the figure. The ' + ' shows the centre of the vessel, $(R, Z)=(175,0) \mathrm{cm}$. at the inner boundary, which are distributed poloidally as a function of distance between neighbouring magnetic flux surfaces, i.e. larger flux at smaller distance and vice versa, implicitly assuming constant temperature and density profiles along the flux surfaces. The energy flux is equally split between ions and electrons. For momentum transport, a zero flux is assumed. At the outer boundary, the decay lengths of the plasma fields (density, temperature, parallel flow) are specified. The outer boundary was chosen sufficiently far away such that the numerical values of the decay length boundary conditions do not affect the edge profiles in a significant way. The volume sources for the particle, momentum and energy balance are also distributed in the computational domain according to the source terms in equations (1)-(4) given by EIRENE, for which the outer boundary has been extended up to the vessel wall. At the wall, the TRIM database reflection model is applied for neutrals with a recycling coefficient of 1. In EMC3 the MC fluid elements are traced by a random walk process with jump steps determined by the transport equations [14], until they are lost at either the outer boundary or the divertor plate, where the Bohm sheath condition is imposed. The recycling flux is taken into account by EIRENE and its distribution on the divertor plate is taken to be proportional to the ion deposition pattern onto the divertor target, with a recycling coefficient of 1 .

In the present computations, the ratio of the vessel wall flux to the divertor flux was estimated to be less than $10 \%$. This will, however, change depending on the plasma decay lengths assumed at the outer surface, which are, in principle, unknown parameters. Because of the recycling coefficients of 1 at all material surfaces and no pumping assumed, the particle throughput is zero in the model. Whether or not an inclusion of the pumping (by ALT limiter) and an external particle source changes the results would depend on several additional physics, i.e. the location of the ALT limiter, the exhaust efficiency and the ergodization effect on the efficiency, which are beyond the scope of our present model.

The computational grids have been constructed mostly taking into account the shape of the divertor plate. Since the divertor plate is almost parallel to the B field lines near the midplane, it was necessary to make the grid finer there to have enough resolution on the divertor surface for computing the deposition pattern. A shallowness of the incident angle of field lines, $\sim 1^{\circ}$ around the midplane, would introduce kinetic effects due to a finite Larmor radius, for which, however, no proper boundary condition exists in terms of fluid description. The expected effects of such shallowness is a broadening of the observed deposition pattern (e.g. figure 9), due to an additional flux via perpendicular transport. A detailed estimation of the effects has to await comparison with experiments. The physical and numerical complications resulting from the shallowness of the incident angle are reduced, to some extent, for the case with the perturbation field turned on, due to the radial and poloidal deflection of the $\mathrm{B}$ lines.

\section{Magnetic field configuration and the effects on radial transport}

The magnetic field configurations for the present computation with the DED field are shown in figure 2, being unfolded into the minor radius, $r$, and the poloidal angle, $\theta$, coordinates. The 

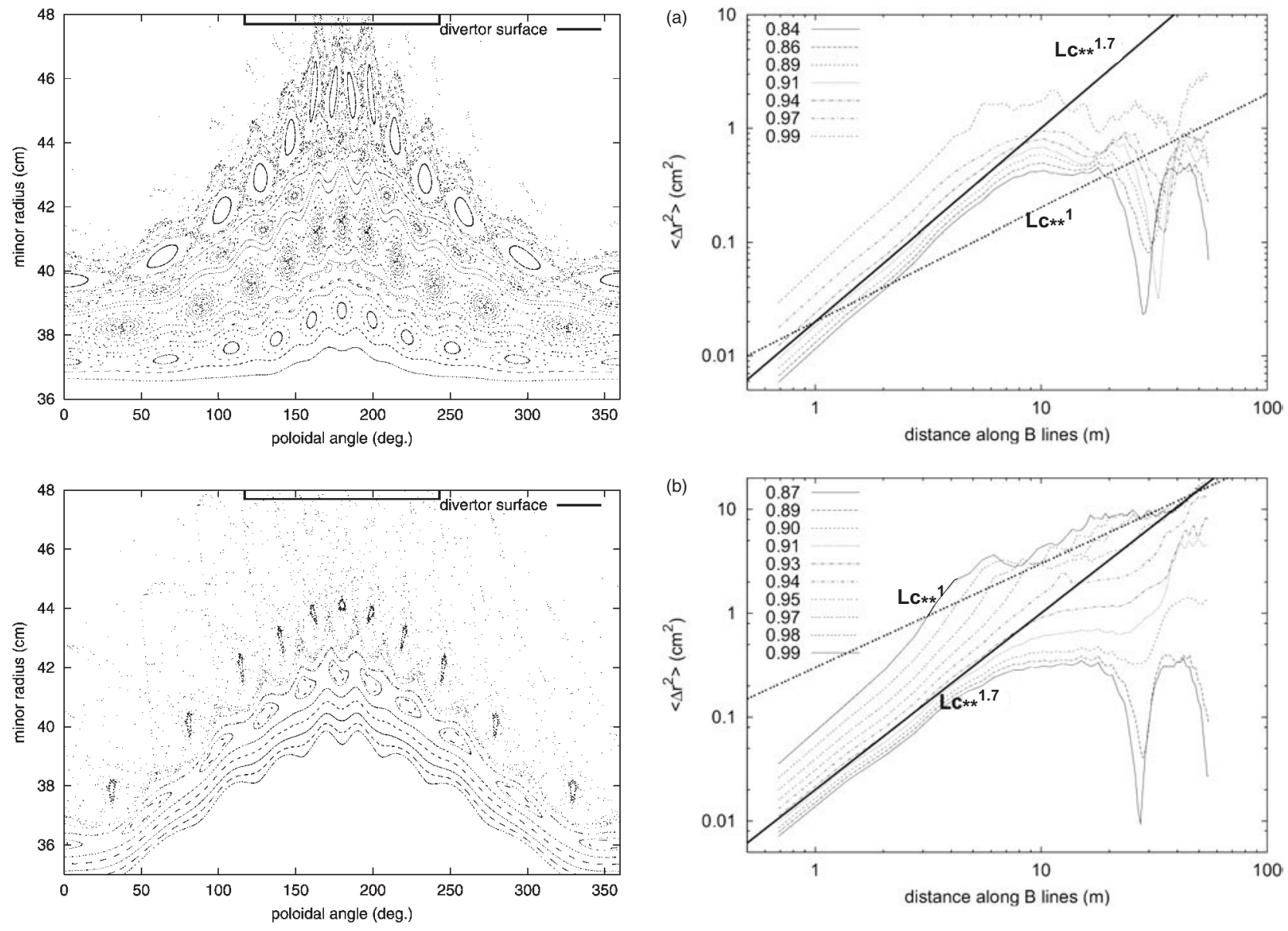

Figure 2. The Poincaré plot at $\phi=0^{\circ}$ with $I_{p}=380 \mathrm{kA}$,

$B_{t}=1.9 \mathrm{~T}, B_{Z}=200 \mathrm{G}, I_{\mathrm{DED}}=15 \mathrm{kA}$, and $\beta_{p}=(a) 1.0$ and $(b)$ 0.0 . The centre of the minor radius is at the centre of the vacuum vessel, $R=175 \mathrm{~cm}, Z=0 \mathrm{~cm}$. The poloidal angle starts from the LFS. The solid line represents the divertor surface at $r=47.7 \mathrm{~cm}$.

angular coordinate $\theta$ starts at the midplane of the low field side (LFS). The various model parameters are taken as follows: toroidal field at the centre of the vessel $B_{t}=1.9 \mathrm{~T}$, plasma current $I_{p}=380 \mathrm{kA}$, poloidal beta $\beta_{p}=1.0$ or 0.0 , DED coil current $I_{\mathrm{DED}}=15 \mathrm{kA}$ and the vertical field $B_{Z}=200 \mathrm{G}$. The resonance surface to the main mode $(m, n)=(12,4)$ is located around $r=44$ and $45 \mathrm{~cm}$ at the HFS for $\beta_{p}=1.0$ and 0.0 , respectively. For the purpose of this study we use (abuse) the value of $\beta_{p}$ as a simple parameter to scan the degree of ergodization in an easy way. In spite of the maximum perturbation strength with $I_{\mathrm{DED}}=15 \mathrm{kA}$ and the inward shift, most of the island chains, e.g. $m=13,12,11$ and 10 , are still visible in the case of $\beta_{p}=1.0$. This is due to the high $\beta_{p}$, which gives rise to a larger Shafranov shift, so that the distance between flux surfaces at the HFS is widened, preventing an overlapping of the island chains. In the case of $\beta_{p}=0.0$, on the other hand, a clear island structure can hardly be found outside of the $m=10$ island chain, this indicating a higher degree of ergodization than in the $\beta_{p}=1.0$ case. The degree of ergodization is measured with the rate of divergence of the neighbouring field lines. In figure 3 we plot the radial deviation of the field line trajectories from the unperturbed equilibrium flux surfaces $\left\langle\Delta r^{2}\right\rangle$, against the distance along the field lines $L_{C}$, for different $\beta_{p}$ values. The numbering of the

Figure 3. The second moment of the radial deviation of the field line trajectories from the unperturbed flux surface vs the distance along B lines, $L_{C}$, for $\beta_{p}=(a) 1.0$ and $(b) 0.0$. The numbering of the curves represents the index of the magnetic flux surfaces, increasing outwards in minor radius. The index ' 1 ' represents the LCFS. For each curve, 1000 field trajectories with the starting points poloidally distributed along the flux surface were averaged. The solid and dashed straight lines show the function of $\left\langle\Delta r^{2}\right\rangle \sim L_{C}^{1.7}$ and $L_{C}^{1}$, respectively.

curves indicates an index of the flux surfaces normalized at the LCFS of the non-perturbative case. For each curve, 1000 trajectories are averaged over the poloidal direction. In the case of $\beta_{p}=1.0,\left\langle\Delta r^{2}\right\rangle$ grows initially with a power of 1.7 of the connection length up to $L_{c} \sim 10 \mathrm{~m}$, then reaches a saturation followed by the steep drop around $L_{c} \approx 30 \mathrm{~m}$, which reflects the island structure and is repeated regularly at $L_{c}>30 \mathrm{~m}$. In this case, the relation $\left\langle\Delta r^{2}\right\rangle \sim L_{C}^{1}$ is found nowhere; with this one would have been able to define an ergodization of field lines by a stochastic field model. In the case of $\beta_{p}=0.0$, on the contrary, after the growth with a power of 1.7 similar to $\beta_{p}=1.0$, there appears a region where $\left\langle\Delta r^{2}\right\rangle$ continues to grow, especially outside the index of 0.90 . This is just outside the $m=10$ island chain. In this region one could roughly recover the relation $\left\langle\Delta r^{2}\right\rangle \sim L_{C}^{1}$, which implies a diffusive behaviour of the field lines. From the initial rapid growth in the figure, one can estimate the Kolmogorov length $L_{K} \sim 10-20 \mathrm{~m}$. If we take the quasi-linear form,

$$
L_{K}=\pi q R_{0}\left(\frac{\pi \sigma_{\mathrm{Chir}}}{2}\right)^{-4 / 3},
$$


it gives $L_{K} \approx 9 \mathrm{~m}$ for $q=3, R_{0}=1.75 \mathrm{~m}$ and $\sigma_{\mathrm{Chir}}=1$. Considering the fact that the quasi-linear expression tends to overestimate the degree of ergodization [17], we shall take the value of $L_{K} \approx 15 \mathrm{~m}$ for our configuration. From the linear regime in figure $3(b)$, the diffusion coefficient of the field lines $D_{\mathrm{FL}}$ is estimated to be $\sim 10^{-5} \mathrm{~m}$. It has to be kept in mind, however, that in this analysis the tracing was continued even when the trajectory hits the divertor surface, in order to quantify a purely topological property of the field lines. In terms of plasma transport, however, the ergodization effects vanish at the very edge close to the divertor target due to the intersection of the field lines with solid surfaces. The laminar zone has just been defined to replace this sub-domain. The effects of the laminar zone will be discussed in section 5 .

The radial profiles of the poloidally averaged electron temperature $\left(T_{e}\right)$ are shown in figure 4 . There is no significant difference between 0 and $15 \mathrm{kA}$ for $\beta_{p}=1.0$, while $T_{e}$ decreases about $10-20 \mathrm{eV}$ for $\beta_{p}=0.0$. This is a clear signature of the effective enhancement of the cross-field transport caused by the ergodization. The reduction of $T_{e}$ around the inner boundary in spite of the no ergodicity there (figure $2(b)$ ), results from the enhanced transport at the very edge, because the profile is determined as a radial integration, $T(r)=\Gamma_{r} \int_{1}^{r} \mathrm{~d} r / \chi_{\mathrm{erg}}(r)+T(1)$, where $\Gamma_{r}$ is a radial heat flux. The effective heat transport

(a)

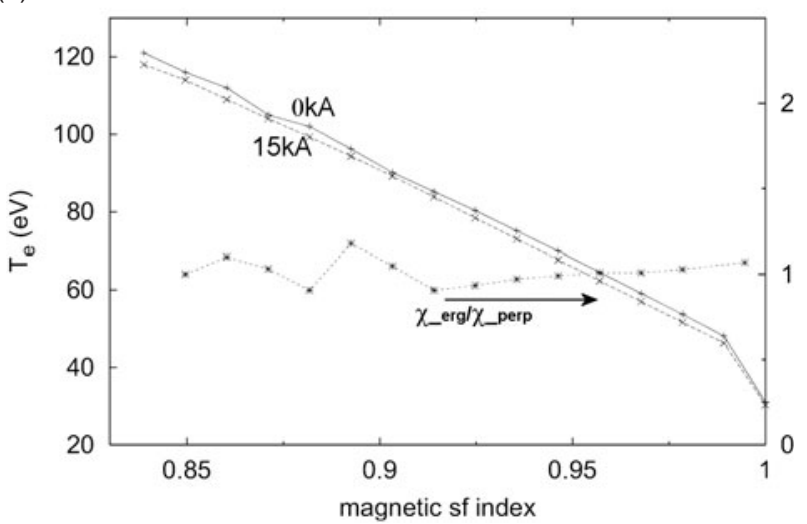

(b)

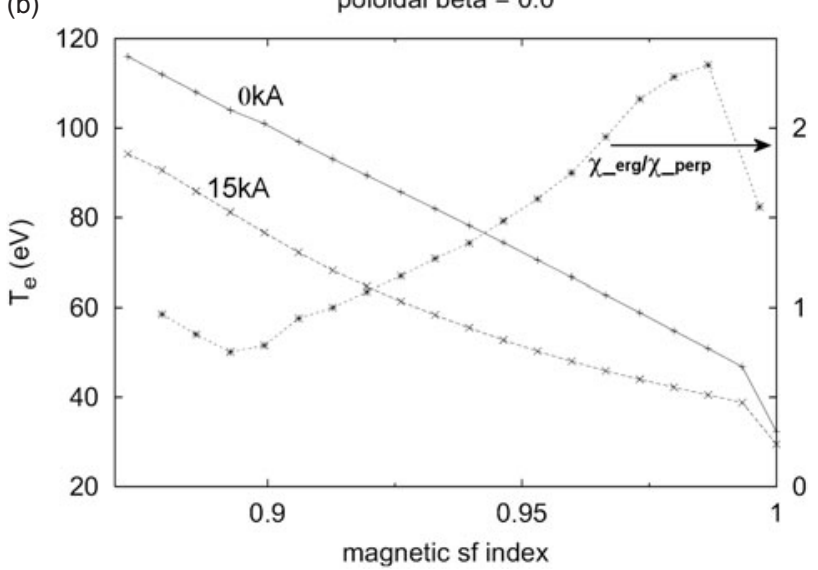

Figure 4. The radial profiles of $T_{e}$ for $\beta_{p}=(a) 1.0$ and $(b) 0.0$, with and without the perturbation field, together with the ratio between the effective cross-field heat diffusivity and the prescribed anomalous value, $\chi_{\mathrm{erg}} / \chi_{\perp}$, calculated from the profiles. $T_{e}$ is averaged along the unperturbed flux surfaces. coefficients, $\chi_{\mathrm{erg}}=\Gamma_{r} /\left(\mathrm{d} T_{e} / \mathrm{d} r\right)$, were calculated from the profiles as shown in the figures, where the enhancement factor was found to be 2.5 at the maximum for $\beta_{p}=0.0$. The quasi-linear model $[3,4]$ predicts the same effect as,

$$
\frac{\chi_{\mathrm{erg}}}{\chi_{\perp}} \approx \frac{\chi_{\|} D_{\mathrm{FL}}}{\chi_{\perp} L_{K}}=\frac{D_{\mathrm{FL}}}{\chi_{\perp} L_{K}} \frac{2.1 \times 10^{22} T_{e}^{2.5}}{n}=8-13
$$

where $\chi_{\perp}$ is the anomalous heat diffusivity. On the other hand, the model by Tokar [18] gives a different expression,

$$
\frac{\chi_{\mathrm{erg}}}{\chi_{\perp}}=\max \left\{\frac{1+\zeta_{T} x \mathrm{e}^{-x}}{1+\zeta_{T} x \mathrm{e}^{-2 x}}\right\}=3.5-4.4,
$$

where $x$ is a distance along field lines normalized by $L_{K}$, $\zeta_{T}=\left(2 D_{\mathrm{FL}} \chi_{\|}\right) /\left(L_{K} \chi_{\perp}\right)$, and it is assumed that the plasma selects the 'optimal' path such that the ratio on the left-hand side of equation (7) takes a maximum value. Compared with the results in figure $4(b)$, the later model seems closer to our results, although ERGOT, applied to Tore Supra-ED conditions, and E3D, applied to TEXTOR-DED conditions showed an agreement with the former one [1,5,8]. Apparently, except for highly idealized problems, the value of this ratio is strongly dependent upon the particular case studied, and probably has to be evaluated on a case-by-case basis from 3D transport models like the one used in this paper. Currently this seems to be the only option in place to separate, experimentally, the unknown anomalous cross-field transport from the terse perturbation field topological effects caused by parallel transport.

In figure 5 we show a re-ionization fraction of the recycling flux from the divertor for $I_{\mathrm{DED}}=0$ and $15 \mathrm{kA}$ in the case of $\beta_{p}=0.0$, where the 3D ionization profiles have been integrated radially inwards, starting from the divertor target. The LCFS for $I_{\mathrm{DED}}=15 \mathrm{kA}$ is deduced to be around the flux surface just outside the $m=10$ islands. It is found that for the perturbative case, a fraction of about $80 \%$ of the recycling flux from the divertor is ionized inside the ergodic region, while for the non-perturbative case about $50 \%$ is ionized outside the LCFS. The remaining fraction not ionized at the edge enters the core plasma and is all ionized there, then comes back to the edge, creating plasma flow. The increased value of the ionization fraction at the edge together with the enhanced

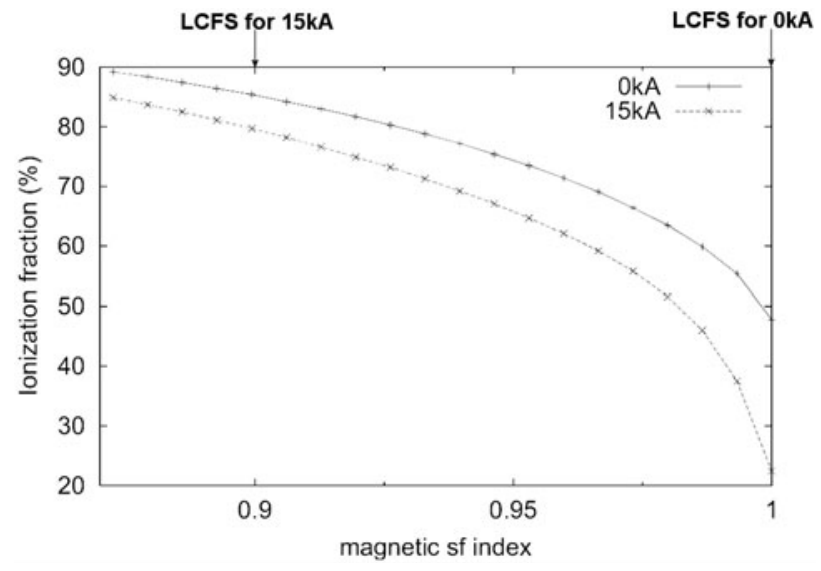

Figure 5. The ionization fraction of the recycling flux from the divertor for $I_{\mathrm{DED}}=0$ and $15 \mathrm{kA}$ in the case of $\beta_{p}=0.0$. 

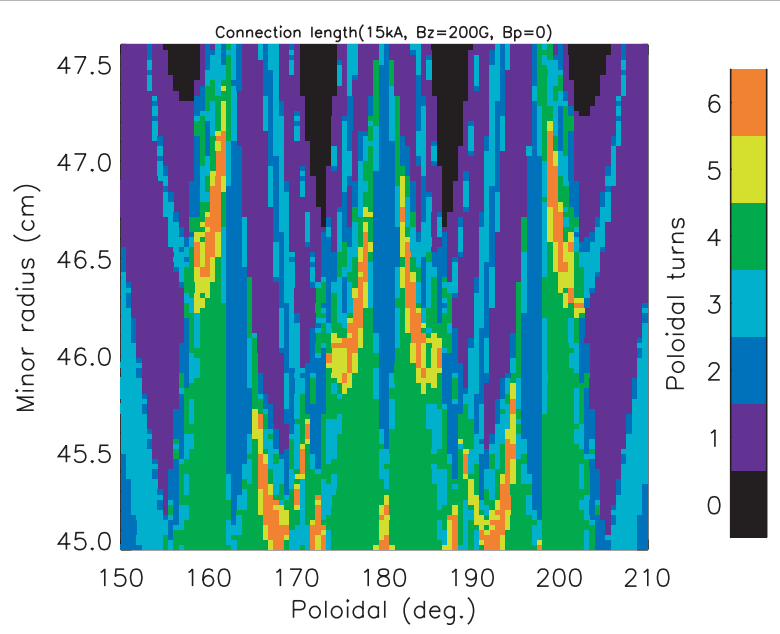

Figure 6. The laminar plot at $\phi=0^{\circ}$ for the $I_{\mathrm{DED}}=15 \mathrm{kA}$ and $\beta_{p}=0.0$. The flux tubes are coloured differently depending on the connection length. One poloidal turn is about $30 \mathrm{~m}$. The figure is resolved up to 6 turns.

radial transport in the perturbative case $\beta_{p}=0.0$ implies the possibility of a transition to a conduction limited regime, i.e. a large temperature drop in a high density operation.

The islands also act to enhance the effective cross-field transport, i.e. a flattening of the radial plasma profiles due to the reconnection of the different flux surfaces results. This is a competition between the cross-field diffusion across the island and the parallel transport along the field lines of the half turn inside the island, and it is most prominent in the $T_{e}$ profile. The effect can be estimated as follows. The perpendicular and parallel transport length in a certain time interval, $\Delta x_{\perp}$ and $\Delta x_{\|}$, may be related with the transport coefficients as

$$
\left\langle\frac{\Delta x_{\perp}}{\Delta x_{\|}}\right\rangle=\sqrt{\frac{n \chi_{\perp}}{\kappa_{\|}}} .
$$

In order for the plasma to see the island, the $\Delta x_{\perp}$ should be much smaller than the island width. To fulfil this condition, one needs a temperature,

$$
T_{e}=\left(\frac{n}{2.1 \times 10^{22}}\left(\frac{L_{\text {island }}}{\Delta x_{\perp}}\right)^{2} \chi_{\perp}\right)^{0.4} \quad(\mathrm{eV}),
$$

where $\Delta x_{\|}$was replaced with the $L_{\text {island }}$, the connection length of a half turn inside the island. With $n=1.0 \times 10^{19} \mathrm{~m}^{-3}$, $\chi_{\perp}=3 \mathrm{~m}^{2} \mathrm{~s}^{-1}, L_{\text {island }}=120 \mathrm{~m}$ and $\Delta x_{\perp}=1 \mathrm{~mm}$ for the $m=11$ island of width $1 \mathrm{~cm}$, it gives $T_{e} \sim 800 \mathrm{eV}$, which is rather difficult to be achieved at the edge region of interest. One also notes that any cross-field convection will smear out the effect. Indeed, inspecting the full 3D model results, we do not observe any significant flattening in the poloidally averaged radial profiles of $T_{e}$. In the case of the other islands ( $m=12$ and 13), it is even more difficult to see such an effect because $L_{\text {island }}$ is longer: $L_{\text {island }}>200 \mathrm{~m}$.

\section{Plasma parameter profiles and magnetic field structure $\left(\boldsymbol{\beta}_{p}=\mathbf{0}\right)$}

In figure 6 we show a so-called 'laminar plot' of $\beta_{p}=0.0$ at the HFS in a poloidal cut, where the flux tubes are
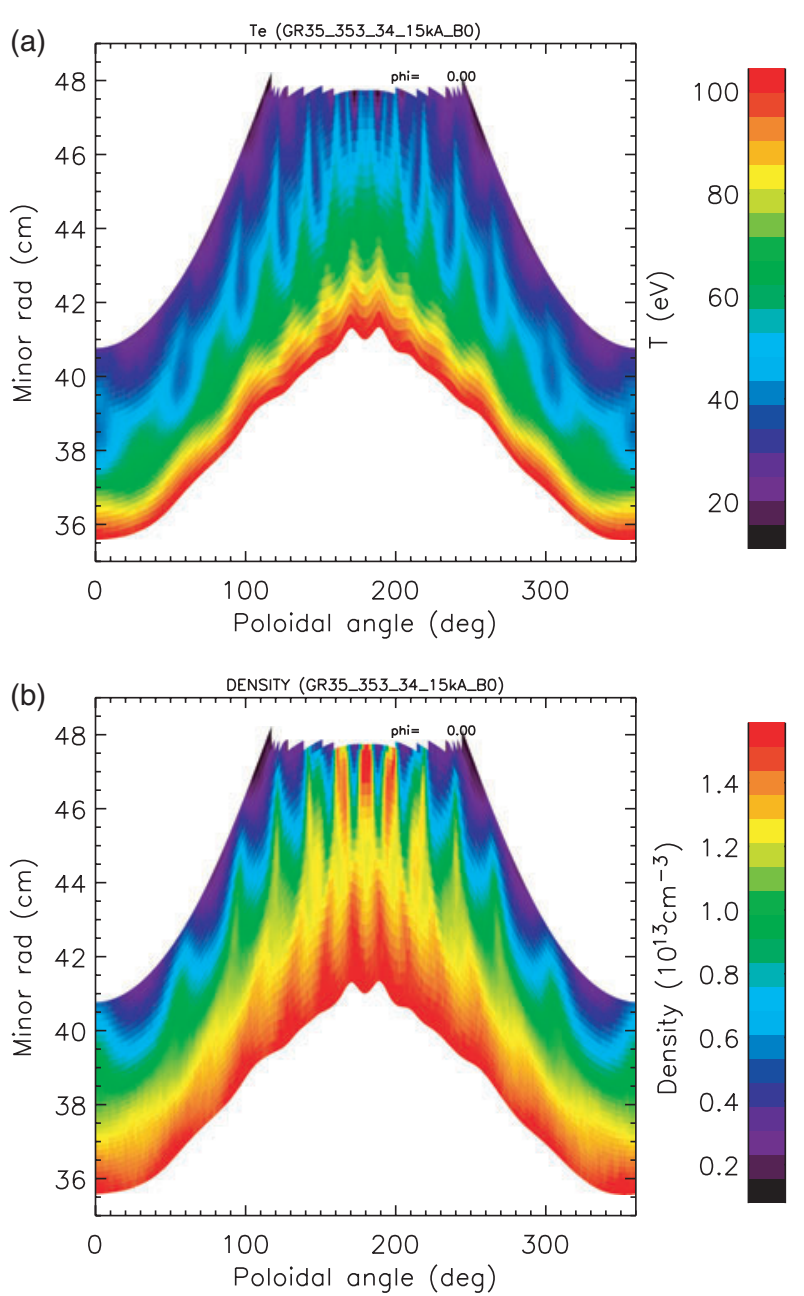

Figure 7. (a) $T_{e}$ and (b) $n$ profiles at $\phi=0^{\circ}$ for the $I_{\mathrm{DED}}=15 \mathrm{kA}$ and $\beta_{p}=0.0$.

coloured differently depending on the connection length. Here the length is normalized to a number of poloidal turns and one poloidal turn is about $30 \mathrm{~m}$ in length. Considering $L_{K} \approx 10-20 \mathrm{~m}$ obtained in the previous section, the ergodic region can be identified with the more-than-four-turn flux tubes. One clearly sees that the transition from the ergodic to the laminar zone has a very complicated surface topology. The very thin fingers of very long flux tubes extending towards the wall are also visible. The pattern is to be compared with the plasma parameter profiles in the poloidal cut of $T_{e}$ and density, $n$ profiles, plotted in figure 7 . The resulting $T_{e}$ profile follows this finger structure closely, because of its very high parallel heat conductivity. This indicates that the fingers provide the loss channel of the energy to the wall. The profile of $n$ also shows a pattern similar to the $T_{e}$ profile but is somewhat more diffused around the fingers, due to the much slower (subsonic convective) parallel transport (as compared with electron heat transport). The density profile is affected by the ionization and convective flow patterns as well, which are shown in figure 8 . The positive value of the Mach number corresponds to a flow in the direction of increasing toroidal angle $\phi$. Six peaks of the ionization source appear in front of the divertor target, these being correlated with the particle deposition pattern, which leads to the increase in density. The two peaks at both edges 

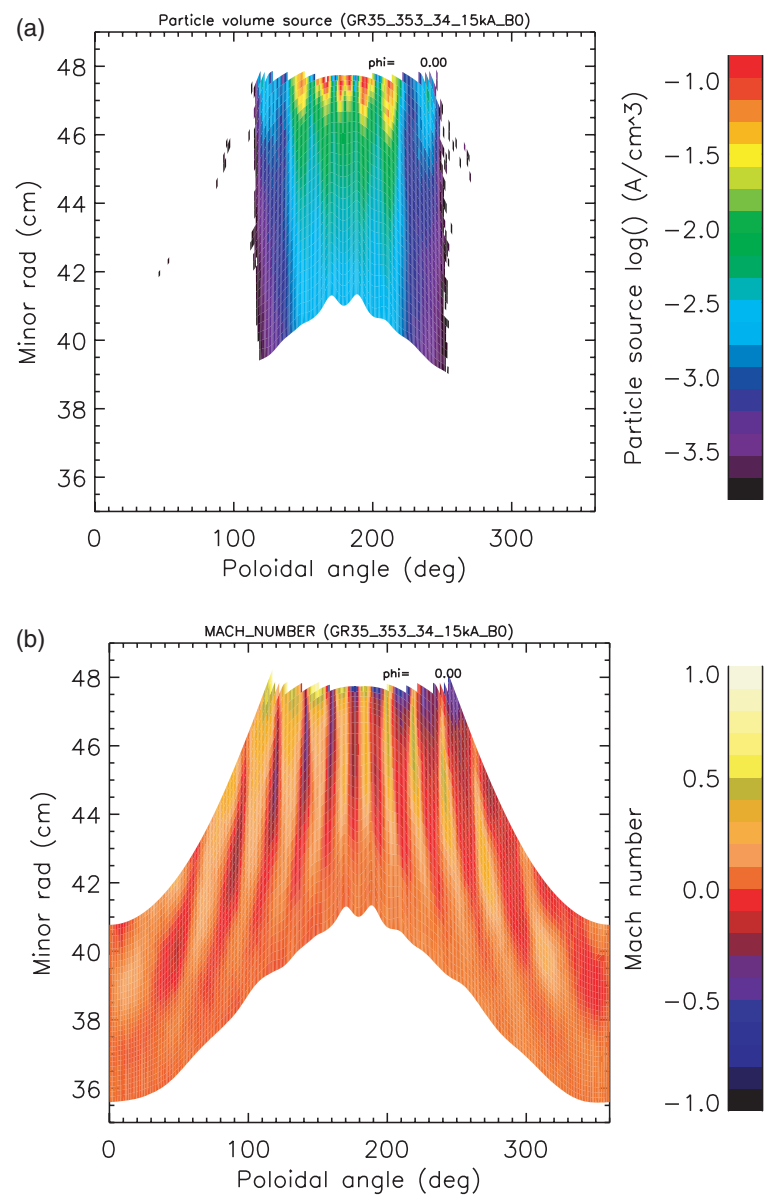

Figure 8. The (a) ionization source and $(b)$ Mach number profile at $\phi=0^{\circ}$ for $I_{\mathrm{DED}}=15 \mathrm{kA}$ and $\beta_{p}=0.0$, with a total recycling flux of $800 \mathrm{~A}$. The positive direction of the Mach number is in the direction of increasing toroidal angle.

$\left(\theta \sim 150^{\circ}\right.$ and $\left.210^{\circ}\right)$ in $n$ are moderate compared to the other three peaks in the centre. This can be understood to be due to the high Mach number (parallel flow) at these places, which pushes the re-ionized plasma towards the divertor, reducing the density there. The Mach number profile has quite a strong poloidal modulation also, but with a pattern different from those of the $T_{e}$ and $n$ profiles. What is quite remarkable is the signature of alternation of the flow direction, which is caused by the direction of the field lines towards and away from the divertor. This might, at higher densities, lead to a parallel momentum loss because of the friction between the counter-streaming flows as already observed in W7-AS [19], both experimentally and in modelling studies. A study of this high density regime (detachment without passing through an intermediate high-recycling regime) is an interesting issue for this configuration, as also mentioned in the previous section, and its accessibility with TEXTOR-DED will have to be investigated in future.

\section{Energy deposition onto the divertor and role of the laminar zone}

As observed in the previous section, the 'fingers' seem to be a major loss channel for the energy and also for the particles. However, at the final phase of the transport towards the divertor,

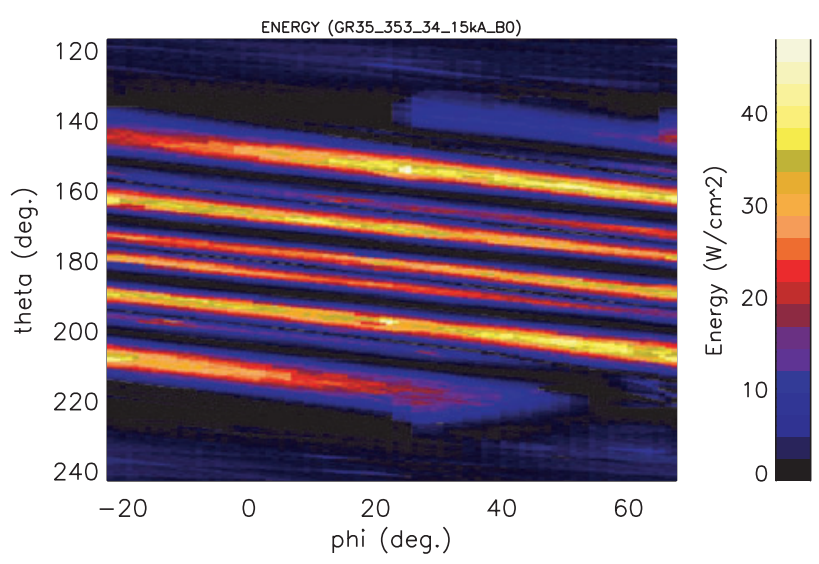

Figure 9. The energy deposition pattern onto the divertor plate for $I_{\mathrm{DED}}=15 \mathrm{kA}$ and $\beta_{p}=0.0$.

a certain amount of energy can also be expected to be given to the shorter flux tubes, i.e. to the laminar zone, by cross-field transport. This is anticipated from figure 6 , where the laminar zone and the ergodic zone can be seen to be interconnected with each other in a complicated manner. The paths of energy flow to targets must, therefore, be a result of a delicate balance at least between parallel transport, cross-field transport and field line geometry. In this section we will investigate the transport process of the energy deposition onto the divertor target by comparing the energy deposition pattern and the connection length profiles on the divertor, which are shown in figure 9 and 10 for $I_{\mathrm{DED}}=15 \mathrm{kA}$. The deposition generally becomes large in between the coils because the radial component of the perturbation field increases there, guiding the flux tubes towards the divertor. The stripe (peak heat load) is found to be split around the midplane, reflecting the splitting of the long connection length flux tube pattern in figure $10(b)$. As for the connection length profiles of figure $10(a)$, because of the weak ergodization in the $\beta_{p}=1.0$ case, most of the area is covered by the one-turn flux tubes that terminate in between the perturbation coils, and the rest is covered by the less-than-oneturn flux tubes (private zone) and by long flux tubes with a very small fraction only ('helical limiter' case). In contrast, in the case $\beta_{p}=0.0$ a substantial area is covered by the longer flux tubes because of the higher degree of ergodization, keeping the global pattern of one-turn and private flux tubes the same as in the $\beta_{p}=1.0$ case. To aid comparison with figure 6 , the laminar plot for $\beta_{p}=1.0$ is shown in figure 11. In these figures, one sees the clear difference between the two cases, i.e. for $\beta_{p}=1.0$ the more-than-six-turn region is bounded by the large one-turn or private flux regions with a very small gap of a few millimetres (filled by the two-to-five-turn region), while for $\beta_{p}=0.0$ the structure is diffusive and not so monotonic in the radial direction as with $\beta_{p}=1.0$. This is a manifestation of the quite different degrees of ergodization, as already shown in section 3 .

The obtained energy deposition patterns onto the divertor were compared with the connection length profiles, figure 10 , and the distribution of energy flux over the flux tubes of different connection length was estimated taking into account the incident angle of the field lines on the divertor surface. The resulting profiles are shown in figure 12. The highest peak appears at $\sim 200 \mathrm{~m}$ (more-than-six-turn) and subsequently at 

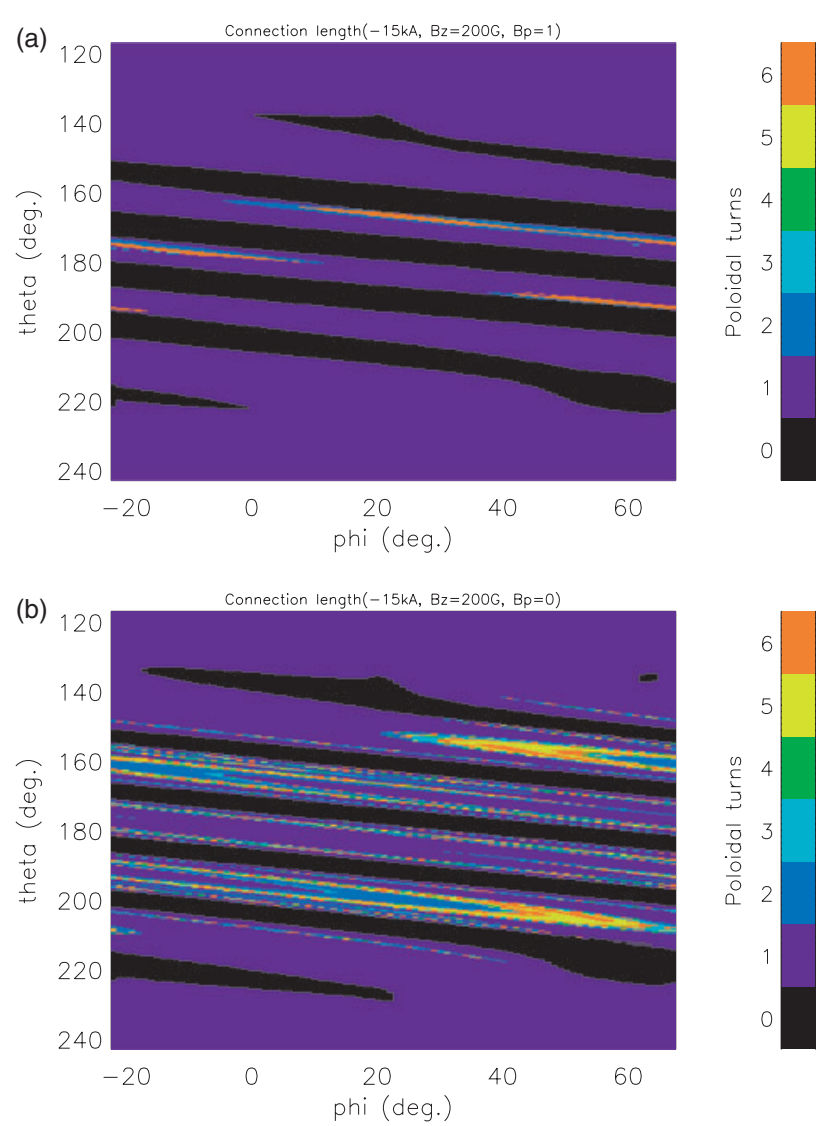

Figure 10. Connection length profiles on the divertor plate for $\beta_{p}=(a) 1.0$ and $(b) 0.0$, being resolved up to six poloidal turns.

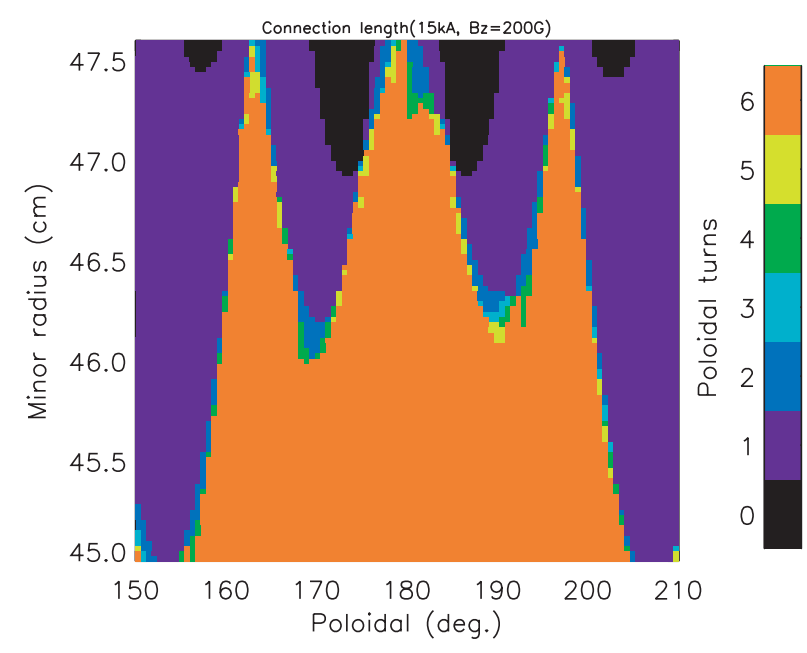

Figure 11. The laminar plot at $\phi=0^{\circ}$ for the $I_{\mathrm{DED}}=15 \mathrm{kA}$ and $\beta_{p}=1.0$.

$100 \mathrm{~m}$ (three-turn), $70 \mathrm{~m}$ (two-turn), $30 \mathrm{~m}$ (one-turn) and the less than $10 \mathrm{~m}$ domain (private flux) in the $\beta_{p}=1.0$ case. This is in accordance with a transport picture, where the long connection length flux tubes provide a major direct loss channel of the energy, as discussed in section 4, and as also already predicted by the earlier E3D simulations [8]; the remaining energy is cascaded down to the shorter flux tubes. One sees, however, that the cascade to the shorter ones does not take place in a way monotonic in the connection length, but instead that the private flux tubes carry an amount of heat comparable to the longer ones (e.g. two-turn). This can be attributed to the particular magnetic field structure for this case shown in figure 11. That is, the two-to-five-turn region is restricted to a very narrow gap of a few millimetres. Hence, the energy coming along the long flux tubes (directly from the ergodic region) can easily be transferred across to the private zone by a perpendicular transport. The collisional mean free path for the parallel electron heat transport, $\lambda_{e}$, is estimated as $\lambda_{e}=\chi_{\|} / v_{\text {the }} \sim 8 \mathrm{~m}$ at the edge region. One thus expects rather effective heat exchange between flux tubes during the flow of energy along field lines of several tens of metres up to the divertor target. It is also to be noted that the shorter flux tubes can act as a sink because of the higher parallel convective flow induced by the Bohm condition at the divertor surface. Additionally, the perpendicular convection might add to the transfer of the energy flux to the private and one-turn flux tubes. In this geometrical analysis, however, one has to keep in mind the following subtlety. The connection length profiles shown in figures 6, 10 and 11 often have a fractal structure [7] and they could, in principle, be resolved arbitrarily finely by increasing the grid resolution of the analysis. The grid of the transport computation, on the other hand, has a finite cell size which is designed to be fine enough to study the fluid equations of interest, i.e. its scale is determined by dissipative effects. This might lead to a contradiction between the results of figures 12 and 10. In figure 10(a), for example, there is only one cell for the five-turn flux tube and several for the four-turn one, where the size of a cell is about $0.6 \mathrm{~cm}^{2}$; but these are so small that the grid of the transport computation does not resolve them. This is the reason why in figure 12(a) there is no peak at four- $(\sim 20 \mathrm{~m})$ and five-turn $(\sim 150 \mathrm{~m})$ flux tubes. There remains indeed a question as to what extent the intrinsically dissipative plasma fluid can resolve such a fine magnetic structure. A natural limit is, certainly, set by the Larmor radii of the particles considered. At the moment, however, and in view of the anyway not identified anomalous cross-field transport properties, we restrict ourselves to study quite a coarse grained picture as outlined above and to identify later, by experiments, if such a picture can be regarded as sufficient.

In the case of $\beta_{p}=0.0$, it is found that the energy is distributed quite uniformly over the entire connection length profile. This is due to the high ergodization level, which makes the field structure even more complicated than in the lower ergodization case (as shown in figure 6). The change of the connection length is not monotonic in the radial direction, and the four-turn-flux tubes dominate at the inner radius rather than more-than-six-turn tubes. Also, the diffusive structure helps to distribute the energy to the various connection length flux tubes. As found in the case of $\beta_{p}=1.0$, the cascade of the energy to the shorter flux tubes is not monotonic in the connection length in this case also.

In figure 13 we show the energy flux distribution normalized with respect to the area of the divertor surface. Because of the low incident angle for longer flux tubes in $\beta_{p}=1.0$, the flux is spread out on the surface as shown in figure 13(a), reducing the peak value, especially at the morethan-six-turn flux tubes. In the analysis of the incident angle, the angle of one-turn was found to be highest. This is the 

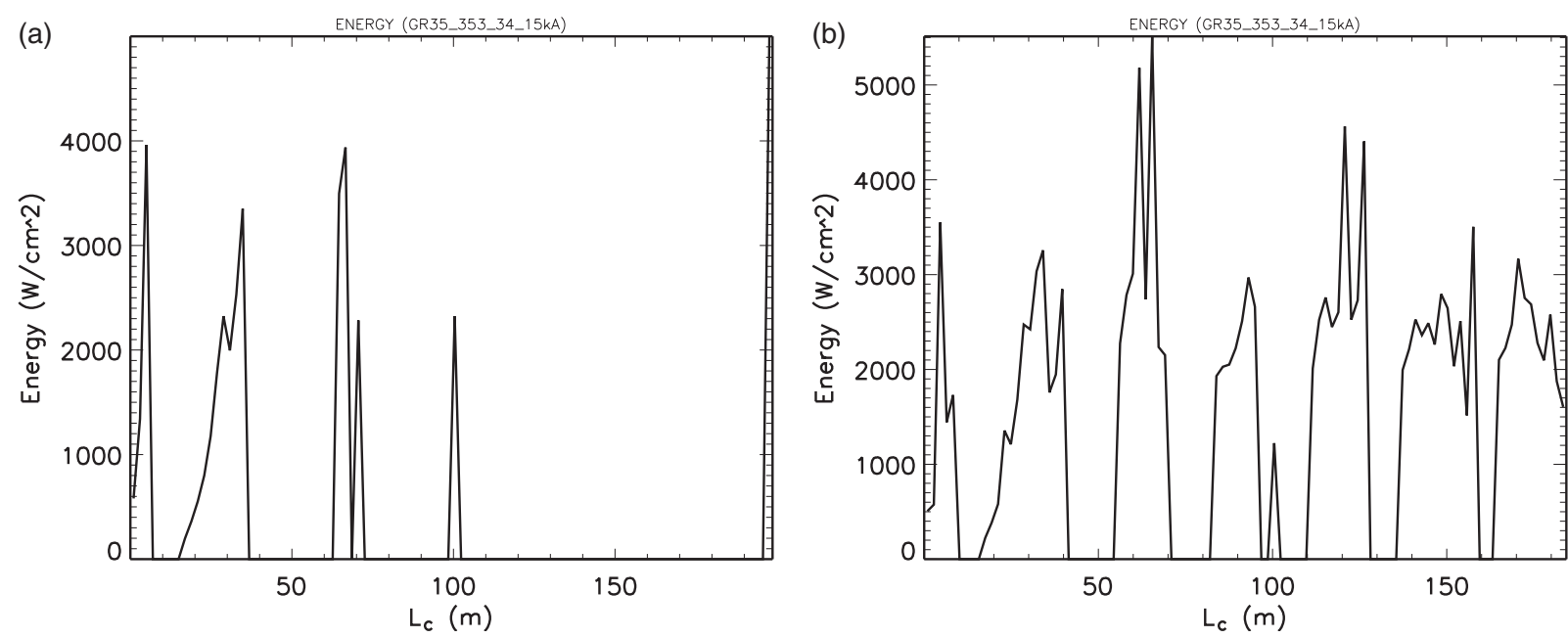

Figure 12. The energy distribution over the flux tubes of different connection length $L_{C}$, for $\beta_{p}=(a) 1.0$ and $(b) 0.0$. The energy flux is normalized with the area perpendicular to the field lines.
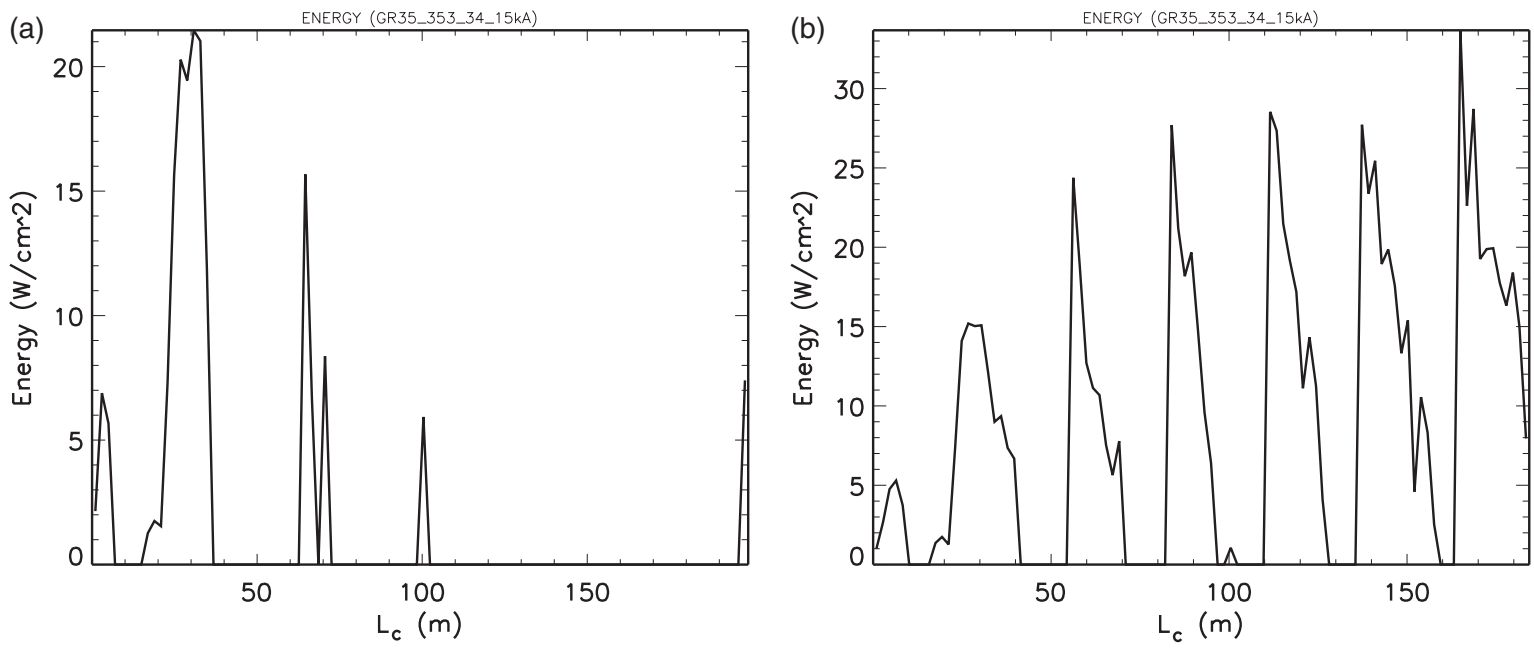

Figure 13. The energy distribution over the flux tubes of different connection length $L_{C}$, for $\beta_{p}=(a) 1.0$ and $(b) 0.0$. The energy flux is normalized with respect to the area of the divertor surface.

reason why the flux of the one-turn becomes high. In the case of $\beta_{p}=0.0$, on the other hand, the flux increases gradually for longer tubes. This is because of the higher incident angle at longer flux tubes, in contrast to the case of $\beta_{p}=1.0$.

As shown in these discussions, the interpretation of mutual influence of the laminar and the ergodic zone on the energy deposition onto the divertor depends on $\beta_{p}$, i.e. the degree of ergodization, and also on the incident angle of the field lines. In terms of the plasma-wall interaction, the high $\beta_{p}$ case is preferable because of its lower energy flux density onto the divertor surface.

\section{Summary}

The transport properties in the TEXTOR-DED configuration have been studied using the 3D plasma edge fluid transport code, EMC3-EIRENE. The code predicts the effects of the DED perturbation field in a static case as follows.

(i) In the case of a high degree of ergodization $\left(\beta_{p}=\right.$ $\left.0.0, D_{\mathrm{FL}} \sim 10^{-5} \mathrm{~m}\right)$, the radial profile of the electron temperature changes significantly, with $\chi_{\mathrm{erg}} / \chi_{\perp} \approx 2.5$. The enhancement of the radial transport for the particular cases studied is closer to the expectation by Tokar's model, which gives $\chi_{\mathrm{erg}} / \chi_{\perp} \approx 3.5$ or less, than to the RechesterRosenbluth model, however: this factor sensitively depends upon various details of the configuration not included in either of these models. Agreement with any such strongly reduced model, therefore, has to be regarded as coincidental at the present stage.

(ii) The recycling flux from the divertor target is ionized up to $80 \%$ outside of the LCFS in the case $\beta_{p}=0.0$, which is higher than in the non-perturbative case by a factor of 1.6. The parallel flow towards the divertor plate gives rise to a momentum loss due to the friction between the counterstreaming flows. These two effects could be important factors that determine the edge transport characteristics, especially in the high density regime not yet investigated for TEXTOR-DED.

(iii) There appears to be a strong poloidal modulation in all parameters, which reflects the connection length profile of the magnetic field. 
(iv) The long flux tubes provide a major loss channel of the plasma to the edge, and the energy flux is partially cascaded down to the shorter ones until deposition onto the divertor. The energy distribution as a function of the connection length significantly depends on the degree of ergodization, i.e. $\beta_{p}$ in our case. In terms of the plasmawall interaction, high $\beta_{p}$ is preferable because of its lower localized heat flux on the divertor surface.

\section{Acknowledgments}

One of the authors, M.K., is grateful to M.Z. Tokar and S.S. Abdullaev for the useful discussions, and for the financial support of the Japan Society for the Promotion of Science, contr. No 2408.

\section{References}

[1] Ghendrih Ph. et al 2002 Nucl. Fusion 421221

[2] Finken K.H. and Wolf G.H. 1997 Fusion Eng. Des. 37337
[3] Rechester A.B. and Rosenbluth M.N. 1978 Phys. Rev. Lett. 4038

[4] Ghendrih Ph. et al 1996 Plasma Phys. Control. Fusion 38 1653

[5] Becoulet M. et al 2000 Contrib. Plasma Phys. 40251

[6] Eich Th. et al 2000 Nucl. Fusion 401757

[7] Abdullaev S.S. et al 2001 Phys. Plasmas 82739

[8] Runov A.M. et al 2001 Phys. Plasmas 8916

[9] Feng Y. et al 2002 Plasma Phys. Control. Fusion 44611

[10] Feng Y. et al 1997 J. Nucl. Mater. 241-243 930

[11] Feng Y. et al 1998 Plasma Phys. Control. Fusion 40371

[12] Reiter D. 1984 Juelich Rep. 1947

[13] Braginskii S.I. 1965 Reviews of Plasma Physics vol 1, ed M.A. Leontovich (New York: Consultants Bureau) p 205

[14] Feng Y. et al 3D edge modelling and island divertor physics Contrib. Plasma Phys. submitted

[15] Kobayashi M. et al Implementation of the EMC3-EIRENE code on TEXTOR-DED: accuracy and convergence study Contrib. Plasma Phys. submitted

[16] Feng Y. and Kisslinger J. 2000 Contrib. Plasma Phys. 40271

[17] Abdullaev S.S. et al 1998 Phys. Plasmas 5196

[18] Tokar M.Z. 1999 Phys. Plasmas 72808

[19] Feng Y. et al 1999 Proc. 26th EPS Conf. on Controlled Fusion and Plasma Physics (Maastricht) vol 23J, p 1465 